\title{
A chemerin peptide analog stimulates tumor growth in two xenograft mouse models of human colorectal carcinoma
}

Justa Friebus-Kardash ${ }^{1,2}$, Petra Schulz ${ }^{1}$, Sandy Reinicke ${ }^{1}$, Cordula Karthaus ${ }^{1}$, Quirino Schefer ${ }^{1}$, Sebastian Bandholtz ${ }^{1}$ and Carsten Grötzinger ${ }^{1,3,4, *}$

1 Department of Hepatology and Gastroenterology, Charité - Universitätsmedizin Berlin, corporate member of Freie Universität Berlin and
Humboldt-Universität zu Berlin, 13353 Berlin, Germany; p.schulz@metriopharm.com (P.S.); sandy.reinicke@posteo.de (S.R.); cordula.kar-
thaus@uni-oldenburg.de (C.K.); quirino.schefer@regenold.com (Q.S.); sbandholtz@gmail.com (S.B.); carsten.groetzinger@charite.de
(C.G.)
Department of Nephrology, University Hospital Essen, University Duisburg-Essen; justa.friebus-kardash@uk-essen.de (J.F.-K.)
$3 \quad$ Partner site Berlin, German Cancer Consortium (DKTK), 13353 Berlin, Germany
$4 \quad$ German Cancer Research Center (DKFZ), 69120 Heidelberg, Germany
* Correspondence: carsten.groetzinger@charite.de

Abstract

Background: Chemerin plasma concentration has been reported to be positively correlated with the risk of colorectal cancer. However, the potential regulation of $\mathrm{CRC}$ tumorigenesis and progression has not yet been investigated in an experimental setting. This study addresses this hypothesis by investigating proliferation, colony formation and migration of $\mathrm{CRC}$ cell lines in vitro as well as in animal models. Methods: In vitro, microscopic assays to study proliferation as well as a scratch-wound assay for migration monitoring were applied using the human CRC cell lines HCT116, HT29 and SW620 under the influence of the chemerin analog CG34. The animal study investigated HCT116-luc and HT29luc subcutaneous tumor size and bioluminescence during treatment with CG34 versus control, followed by ex-vivo analysis of vessel density and mitotic activity. Results: While proliferation of the three CRC cell lines in monolayers was not clearly stimulated by CG34, the chemerin analog promoted colony formation in three-dimensional aggregates. An effect on cell migration was not observed. In the treatment study, CG34 significantly stimulated both growth and bioluminescence signal of HCT116-luc and HT29-luc xenografts. Conclusions: The results of this study represent the first indication of a tumor growth-stimulating effect of chemerin signaling in CRC. 


\section{Introduction}

Chemerin is a regulatory adipokine involved in energy metabolism, regulation of immune function and other physiolog-

ical processes such as chemotaxis, differentiation and proliferation. It exerts its action via its receptors CMKLR1, GPR1

and CCRL2 [1,2]. Both clinical as well as animal studies have clearly demonstrated that secretion and systemic levels of chemerin rise with overweight and adiposity, and decline after diet, exercise-based weight loss, and bariatric surgery [3-6]. Chemerin has been shown to promote chemotaxis of macrophages and NK cells and to enhance migration of plasmacytoid dendritic cells [7-9]. As a chemoattractant in inflammation, chemerin was reported to exert action on NF$\mathrm{KB}$ signaling, thereby promoting pro-inflammatory, but also anti-inflammatory activities [10-12]. In hypertension, chemerin protein levels are elevated, and experimental studies support role of chemerin in the control of blood pressure, while inhibition of angiotensin-l-converting enzyme lowers chemerin serum levels [13-17].

Chemerin has also been implicated in several functions of tumor development, such as growth, invasion, metastasis, immune cell recruitment, and angiogenesis $[18,19]$. In a seminal paper, Pachynski et al. found chemerin to suppress melanoma by recruiting natural killer cell antitumor defenses [20]. Expression of bioactive chemerin by keratinocytes was shown to inhibit late stages of tumor development in a chemical model of skin carcinogenesis [21]. Similarly, chemerin was found to have a protective role in hepatocellular carcinoma (HCC) exerted by inhibiting the expression of IL-6 and GM-CSF, and to suppress HCC metastasis through a CMKLR1-PTEN-Akt axis $[22,23]$. In HCC patients, reduced expression of chemerin is associated with a poor prognosis and a lowed infiltration of both dendritic cells and natural killer cells [24]. While antitumor activity of chemerin has been reported from a variety of cancer types, there are also reports about tumor-promoting effects. Inhibition of the chemerin/CMKLR1 axis in neuroblastoma cells was demonstrated to reduce clonogenicity and cell viability in vitro and to impair tumor growth in vivo [25]. Likewise, chemerin acts via CMKLR1 and GPR1 to stimulate migration and invasion of gastric cancer cells [26].

Regarding colorectal carcinoma (CRC), chemerin plasma concentration was found to be associated with the risk of incident CRC and to be independent of established CRC risk factors $[27,28]$. In addition, serum chemerin levels turned out to be positively associated with the presence of colorectal adenoma in men [29]. Furthermore, chemerin receptor CMKLR1 was detected at higher abundance in CRC tumor tissues than in margins [30].

So far, however, the potential regulation of CRC tumorigenesis and progression has not been investigated in an experimental setting. Recently, our group has reported the development of the metabolically stable high-affinity chemerin peptide agonist CG34 that potently activates both CMKLR1 as well as GPR1. Chelator-linked and radionuclide-loaded CG34 was able to detect via PET/MRI imaging breast cancer in a mouse model that used xenografts of the endogenously CMKLR1-expressing human cell line DU4475 [31]. 
In this study, the chemerin analog CG34 was used to investigate the possible regulation of three human CRC cell line models in vitro with regard to proliferation, colony formation and migration. In addition, CG34 was utilized to study the influence of chemerin receptor signaling on growth and vascularization in two human CRC cell xenograft mouse models. The results of this study represent the first indication of a tumor growth-stimulating effect of chemerin signaling in CRC.

\section{Materials and Methods}

\subsection{Reagents and cell culture}

The luciferase-expressing cell lines HCT116-luc and HT29-luc were purchased from Caliper (Hopkinton, USA). The cell line SW620-luc cell line was created by transduction in our laboratory. All other cell lines were purchased from ATCC/LGC Standards (Wesel, Germany) or CLS (Eppenheim, Germany). The cell lines were cultured in RPMI 1640 medium (Biochrom AG, Berlin, Germany) or McCoy's5A modified medium (Biochrom AG, Berlin, Germany), each supplemented with $10 \%$ fetal calf serum (Biochrom AG, Berlin, Germany). All tumor cell lines were cultured in an incubator (Labotect, Rosdorf, Germany) providing a humidified atmosphere at $37{ }^{\circ} \mathrm{C}$ with $5 \% \mathrm{CO}_{2}$. Cells were passaged every 23 days.

\subsection{RNA isolation, transcription and RT-qPCR}

The preparation of RNA for RT-qPCR experiments was performed according to the manufacturer's instructions using the RNeasy Plus Mini Kit (Qiagen, Hilden, Germany). Isolated RNA was transcribed into cDNA using the High Capacity cDNA Reverse Transcription Kit (Applied Biosystems, Darmstadt, Germany). For this, $3.2 \mu \mathrm{g}$ of RNA was transcribed into $80 \mu \mathrm{L}$ of cDNA (final concentration: $40 \mathrm{ng} / \mu \mathrm{L}$ ) according to the manufacturer's protocol. In addition, as a negative control for each sample, a preparation without reverse transcriptase was analyzed in RT-qPCR. Quantitative real-time Primer sample sets for TaqMan amplifications were obtained from Life Technologies (Darmstadt, Germany). Here, the housekeeping genes were labeled as endogenous controls with the dye VIC and the genes to be analyzed were labeled with FAM. The Fast Start TaqMan® Probe Master Kit (Roche) additionally contains Taq DNA Polymerase, reaction buffer and nucleotides (dATP, dCTP, dGTP, dUTP). PCR was performed using the CFX96 real-time system (Bio-Rad, Düsseldorf, Germany). For this purpose, $30 \mathrm{ng}$ of cDNA were used. The analysis was performed using qBase PLUS (Biogazelle, Zwijnaarde, Belgium) according to the $\Delta \Delta$ Ct-method. As multiple reference genes were included in the normalization, the geometric mean of the relative housekeeping expression was first determined and then this value was used to normalize the target gene expression. Subsequently, these data were normalized to a cq value of 34 .

\subsection{Chemerin ELISA}


used. Cells were incubated in full media at a density of approximately $70 \%$ for 24 hours; supernatants were immediately used for ELISA or frozen at $-80^{\circ} \mathrm{C}$. The ELISA was performed according to the manufacturer's protocol with the supplied standards. MaxiSorp 96-well plates (Nunc, Thermo Fisher Scientific, Dreieich, Germany) were used. The measurement was performed in the SpectramaxPlus384 reader (Molecular Devices, Biberach an der Riß, Germany) at $650 \mathrm{~nm}$ (before addition of the stop solution) or $450 \mathrm{~nm}$ (after addition of the stop solution) and the concentration was determined based on the standard curve using SoftMax Pro 5.3.

\subsection{Proliferation assay using DAPI staining of nuclei}

After reaching a confluence level of $60-70 \%$, cells were trypsinized (trypsin/EDTA solution, Biochrom AG, Berlin, Germany) and seeded onto a 96-well plate with transparent, microscopy grade bottom (Becton Dickinson, Heidelberg, Germany). Cell seeding was then performed by pipetting $100 \mu$ l containing 10,000 cells per well.

At $1 \mathrm{~h}$ after cell seeding, the substances to be tested, including negative controls (medium containing $1.6 \%, 0.8 \%$ or 0.6\% FCS for HCT116-luc, HT29-luc and SW620-luc, respectively, and positive controls (medium containing 10\% FCS) were added to the cells in a volume of $100 \mu \mathrm{l}$ per well. The chemerin analog CG34 (final concentration $10 \mu \mathrm{M}$ ) was diluted in the medium corresponding to the negative controls, as stated above. To avoid loss of substance activity over time, media with or without compound was exchanged every 24 hours.

After 96 hours, cells were fixed for 10 minutes in a PBS-buffered 4\% PFA solution (Herbeta Arzneimittel, Berlin, Germany) pipetted directly into the medium. Then, cells were subjected to $10 \mathrm{~min}$ of incubation with $5 \mu \mathrm{g} / \mathrm{ml}$ DAPI (SigmaAldrich, Munich, Germany) and 0.1\% Triton (Merck, Darmstadt, Germany) in PBS. Plates were washed 2 times with PBS. After the last wash, PBS was left in the plate. The plate was covered with a transparent foil and stored at $4^{\circ} \mathrm{C}$ until measurement. The number of nuclei was determined using high-content analysis with the IN Cell Analyzer automated microscope (GE Healthcare, Reading, UK). Data are reported as relative cell numbers in \% of the positive control.

\subsection{Proliferation assay using bright-field microscopy of growing cells}

After reaching a confluence level of $60-70 \%$, cells were trypsinized and seeded onto a 96 -well standard cell culture plate (Sarstedt, Nümbrecht, Germany). Cell seeding was then performed done by pipetting $100 \mu \mathrm{l}$ each with 10,000 cells per well. Directly after cell seeding, $100 \mu \mathrm{l}$ per well of RPMI1640 with 10\% FCS containing the chemerin analog CG34 were added to the cells. As negative controls, other wells were incubated in RPMI1640 with $10 \%$ FCS only. Plates were then placed for up to five days into an automated microscopy system (Incucyte S3, Essen Bioscience, Göttingen, Germany). Bright-field images of each well were taken every two hours. To avoid loss of substance activity over time, media with 
an adapted segmentation algorithm. Using kinetic confluency data, the rate constant $k$ for cell growth up to three days after seeding was calculated using the exponential growth equation $\left(Y=Y 0^{*} \exp \left(k^{*} X\right)\right.$ in GraphPad Prism 7.0 (GraphPad Software, San Diego, USA).

\subsection{Colony formation assay}

Cells were seeded in a flask and incubated for 3 days until reaching $70 \%$ confluence. Cells were then trypsinized, suspended in serum-free RPMI1640 medium, and counted using a Neubauer counting chamber. Four ml of a cell suspension containing 30,000 cells per $1 \mathrm{ml}$ of FCS-free medium were prepared. In parallel, a sterile $\beta$-mercaptoethanol solution was prepared (5 $\mu \mathrm{l}$ of 2-mercaptoethanol (Sigma-Aldrich, Munich, Germany)) in $8.45 \mathrm{ml}$ of PBS. In addition, methylcellulose aliquots (Sigma-Aldrich, Munich, Germany) were pre-warmed at $37^{\circ} \mathrm{C}$. Under sterile conditions, a mixture of $3.6 \mathrm{ml}$ of methylcellulose, $2.7 \mathrm{ml}$ of FCS, $60 \mu \mathrm{l}$ of mercaptoethanol solution, $770 \mu \mathrm{l}$ of Iscove's medium (Biochrom AG, Berlin, Germany) and $300 \mu \mathrm{l}$ of the cell suspension was prepared in a tube. The tube was shaken vigorously for approximately $5 \mathrm{~min}$ until the preparation took on a pink color. Then, $1.63 \mathrm{ml}$ of agar previously boiled in the microwave and dissolved in the serum-free medium were added to the batch, shaken vigorously repeatedly, and placed in the cell incubator for $20 \mathrm{~min}$. After the incubation period had elapsed, $1 \mathrm{ml}$ of the resulting cell mixture was spread evenly on a gridded $3.5-\mathrm{cm}$ cell culture dish using an insulin syringe, taking care to prevent the formation of air bubbles. For both CG34 treatment as well as the media control, three small cell culture dishes $(2 \mathrm{~mm}$ grid, Nalge Nunc International, Rochester, USA) were filled with the cell preparation and placed in a large cell culture dish, which was previously loaded with a small dish filled with PBS to prevent the preparation from drying out. Next day, each cell batch was treated with $100 \mu \mathrm{l}$ of the substance to be tested for stimulation of colony formation. The negative control consisted of the equivalent treatment with $100 \mu \mathrm{l}$ of medium containing a concentration of FCS specified for the cell line under investigation (see DAPI assay). CG34 was diluted (100 nM) in the medium of the FCS concentration corresponding to the negative control. Subsequently, compound or negative control additions were made once daily, with only $10 \mu$ added to each batch. The large cell culture dishes containing the preparations were incubated in an incubator for 7 days. On the last day of the experiment, tumor cell colonies were manually counted at a defined size of at least 20 cells per colony under a brightfield microscope at 40x magnification. Data are reported as relative cell number in $\%$ of the negative control.

After reaching a confluence level of $80-90 \%$, cells were trypsinized and seeded in a 96 -well standard cell culture plate (Sarstedt, Nümbrecht, Germany). Cell seeding was then performed done by pipetting $100 \mu \mathrm{l}$ of RPMI1640 with $10 \%$ 
once with medium. Thereafter, $200 \mu \mathrm{l}$ per well of RPMI1640 with 10\% FCS containing the chemerin analog CG34 (10 $\mu \mathrm{M}$ ) was added to the cells. As negative controls, other wells were incubated in RPMI 1640 with $10 \%$ FCS only. Plates

\subsection{Treatment study}

To analyze potential tumor growth effect of CG34 in vivo, a treatment study was performed. Two luciferase-expressing xenograft mouse models for human colorectal carcinoma were used: HCT116-luc and HT29-luc. 12 female 8-10 weeks old NMRI nude mice were each implanted subcutaneously with $5 \times 10^{6}$ cells in $100 \mu$ IPMI1640 each of the HT29-luc (left flank) or HCT116-luc (right flank). Tumor growth was monitored once weekly from the first day of the experiment using both caliper size measurements and bioluminescence imaging (IVIS Lumina, Caliper (Hopkinton, USA). On the seventh day, the 12 animals were randomized into two groups with six animals per group. Treatment was started on day eight of the experiment and terminated on day 28. Treatment was administered in a blinded manner (the investigators did not know the assignment of groups until after evaluation), with one group receiving intraperitoneal injections of $200 \mu \mathrm{l}$ of CG34 $(50 \mathrm{nmol})$ per mouse once daily and the other group receiving equivalent treatment with $200 \mu \mathrm{l}$ of water.

After measuring length and width of the tumors with a caliper, volume was calculated according to the formula $\mathrm{v}=0.5^{*}\left(\right.$ length $^{*}$ width $\left.^{2}\right)$.

For bioluminescence imaging, each mouse received an intraperitoneal weight-adapted injection of D-luciferin (150 $\mu$ l)

at a dose of $150 \mu \mathrm{g} / \mathrm{g}$ mouse 5-10 min before imaging. Mice were then placed under isoflurane anesthesia and trans-

ferred to the imager, where bioluminescence signal detection took place. The exposure time was 1 min at the beginning for small tumors and would be reduced to a minimum of 10 seconds as the tumor size increased during the experiment.

The Living Image 3.1 software (Caliper (Hopkinton, USA) was used to count the emitted photons, ultimately determining the photon yield in a region of interest corresponding to the tumor under investigation. In addition, animal weight was monitored twice a week. At the designated experimental endpoint, day 31, animals were anesthetized with isoflurane and euthanized by cervical dislocation. The endpoint tumor volume was determined using a caliper of tumors on sacrificed mice (length, width, and height at autopsy). In addition, the weight of the tumors obtained at autopsy was determined. The excised tumors were frozen in liquid nitrogen and stored at $-80^{\circ} \mathrm{C}$. 


\subsection{CD31 histology}

To visualize tumor vascularization, immunohistochemical staining was performed using an antibody directed against the

fixed with 4\% PFA solution for $20 \mathrm{~min}$ at room temperature. To block endogenous tissue peroxidases and prevent second step, the biotinylated rabbit anti-rat secondary antibody (DAKO) was applied. After rinsing the sections with (50 mM acetate buffer, pH 5.0), AEC solution (Sigma-Aldrich, Munich, Germany), $30 \% \mathrm{H}_{2} \mathrm{O}_{2}$ ) was pipetted onto the sections. The gradual red staining of mouse endothelial cells was followed under the microscope and stopped after 10 min by adding distilled water. Counterstaining with hematoxylin for 10 min was performed to better assess tissue structure. To determine the number of vessels in the tumors, 3 to 4 field-of-view images were taken per tumor preparation using a bright-field microscope at $200 \times$ objective magnification. In each field of view, the red-stained vessels were counted using ImageJ v145.

\subsection{Ki67 histology}

To determine the fraction of proliferating tumor cells, immunohistochemical staining was done with an antibody against human Ki67 (M7240, DAKO). $10 \mu$ m-thick tumor cryosections were fixed with a 4\% PFA solution. Further treatment of the slides was performed according to the protocol already described for CD31 staining. The anti-human Ki67 antibody was applied to the sections at a concentration of $0.0016 \mathrm{mg} / \mathrm{ml}$. The primary antibody was pretreated with a biotinylation reagent (Animal Research Kit, DAKO, Denmark) for $20 \mathrm{~min}$. In addition, the primary antibody was mixed with a blocking reagent (DAKO) for $20 \mathrm{~min}$ before administration to the cryosections. After incubation overnight at $4{ }^{\circ} \mathrm{C}$, the avidin-biotin complex was applied to the tumor tissue sections for $30 \mathrm{~min}$, which were later developed with AEC. The applied staining reaction was stopped after complete red staining of the proliferating Ki67 containing tumor cells using distilled water. Counterstaining with hematoxylin was used to improve identification of the non-proliferating tumor cells. Counting of 


\section{Results}

3.1 Expression of chemerin, CMKLR1, GPR1 and CCLR2 in CRC and other cancer cell lines

To characterize the expression of the primary molecules involved in chemerin signaling in colorectal carcinoma (CRC)

and other cancer cell lines, RT-qPCR was performed on cDNAs from such cells. Five CRC and 35 other cell lines,

including a CMKLR1-overexpressing HEK293 cell clone, were tested for mRNA levels of CMKLR1, GPR1, CCRL2 and 
bioRxiv preprint doi: https://doi.org/10.1101/2021.11.19.469262; this version posted November 20, 2021. The copyright holder for this preprint (which was not certified by peer review) is the author/funder, who has granted bioRxiv a license to display the preprint in perpetuity. It is made available under aCC-BY-NC-ND 4.0 International license.

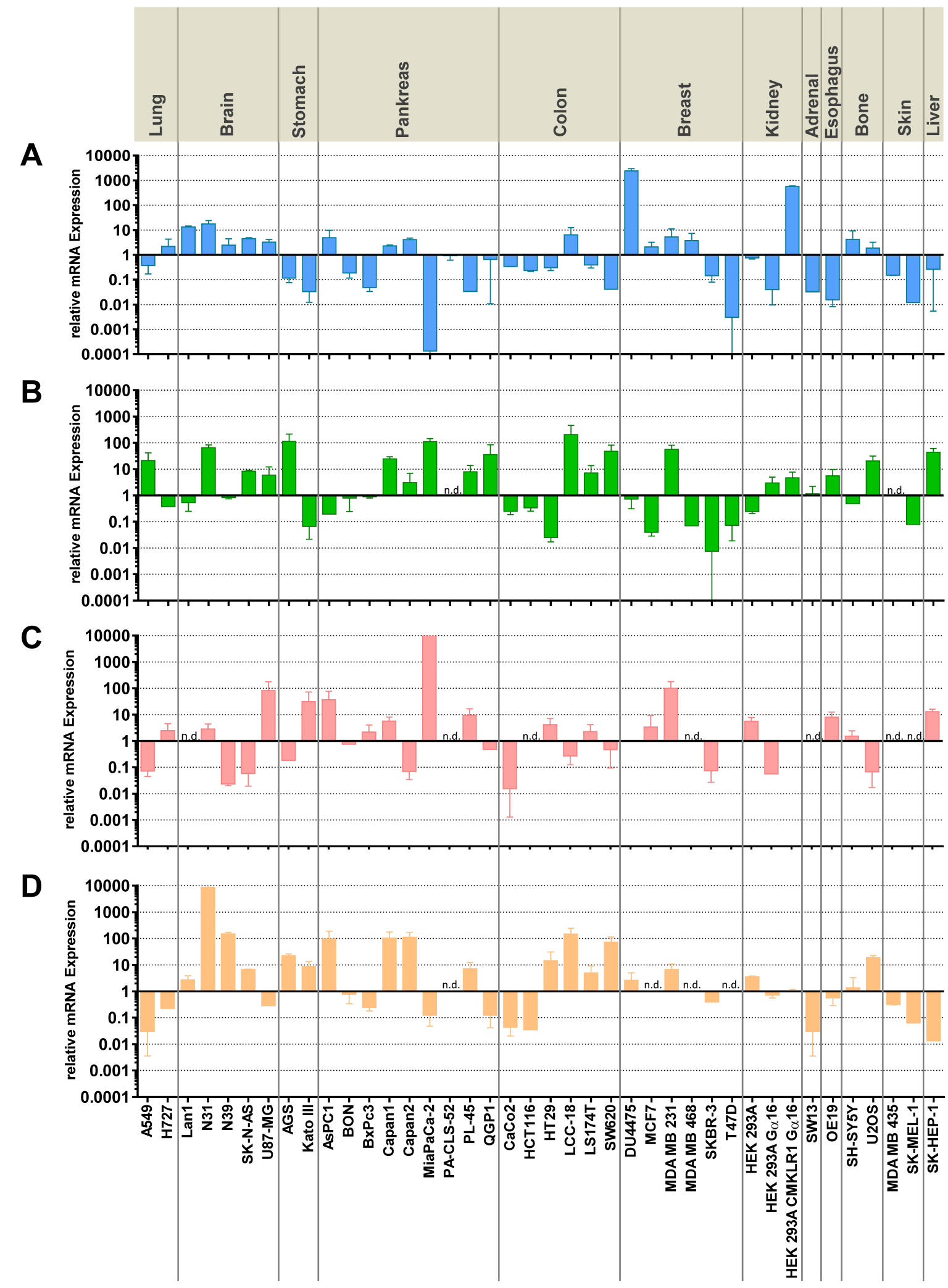

Figure 1. Expression of CMKLR1, GPR1, CCRL2 and chemerin (RARRES2) mRNA in human colorectal and other cancer cell lines as determined by quantitative RT-qPCR. (A) CMKLR1; (B) GPR1; (C) CCRL2; (D) chemerin (RARRES2). The upper section denotes the organ of tumor origin; cell line designations are provided at the bottom. Data have been normalized to reference genes UBC, ALG9 and GAPDH. A value of 1 in the graph corresponds to a cq of 34 . All data represent mean \pm S.E.M. of $n=2-5$ independent 


\subsection{Secretion of chemerin from colorectal and other cancer cell lines}

To verify whether mRNA expression of RARRES2 correlates with the amount of secreted chemerin, supernatants from

33 cell lines were analyzed after 24 hours of culture using a chemerin-specific ELISA. Secreted chemerin levels above

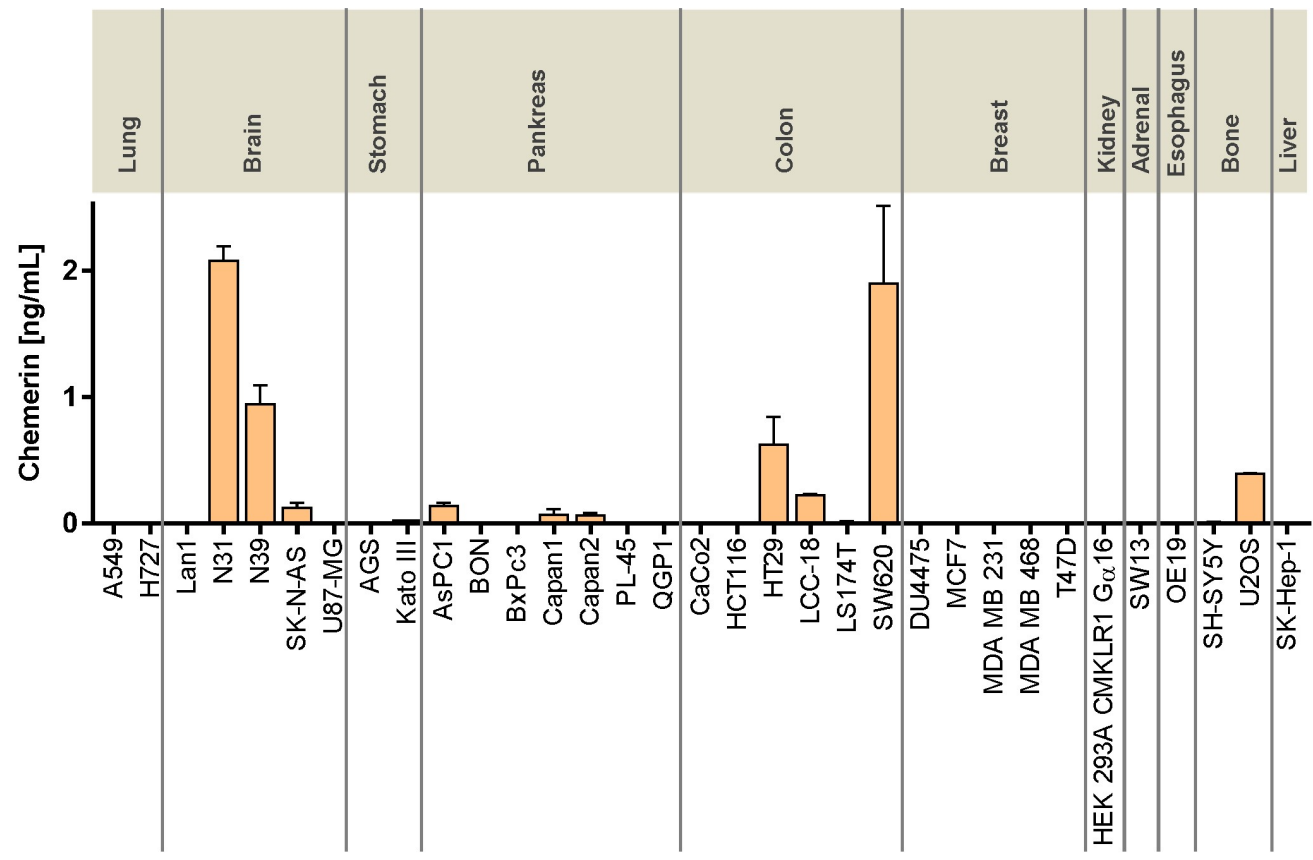

Figure 2. Secretion of the peptide chemerin by CRC and other cancer cell lines as determined by ELISA quantitation of cell culture supernatants. Cells had been cultured at a density of $\sim 70 \%$; supernatants were collected after $24 \mathrm{~h}$ of incubation. The upper section denotes the organ of tumor origin; cell line designations are provided at the bottom. All data represent mean \pm SD of $n=2-6$ independent experiments performed in duplicate.

After having established mRNA levels for chemerin and its receptors as well as secreted amounts of chemerin in culture, secretion, and SW620 expresses higher levels of GPR1, moderate to low levels of the other two receptors, but releases high amounts of chemerin peptide. These three cell lines were investigated for a potential influence of chemerin signaling on three relevant tumor cell characteristics: proliferation, colony formation and migration. As a continuation in an animal model was anticipated, luciferase-transduced cells (HCT116-luc, HT29-luc and SW620-luc) were used in these in vitro 
metabolically stabilized chemerin peptide analog CG34, a potent agonist of CMKLR1 and GPR1 [31], was utilized in all following experiments.

\subsection{Influence of the chemerin analog CG34 on in vitro proliferation, colony formation, migration}

In a first approach, the impact of the chemerin analog CG34 on proliferation of the three CRC cell lines was studied.

Two independent experimental approaches were taken: a high-content analysis quantitation after DAPI-mediated visu-

alization of cellular nuclei (Figure $3 \mathrm{~A}-\mathrm{D}$ ) and a real-time kinetic live cell microscopy approach (Figure 3E-H). In the nuclei-counting experiments, medium with low concentrations of FCS (0.6-1.6\%) were employed as a negative control condition, while cells incubated in medium with 10\% FCS constituted positive controls with maximum stimulation of proliferation. The chemerin analog CG34 only slightly stimulated growth; the difference was not statistically significant in an ANOVA analysis (Figure 3 B-D). Growth of the three CRC cell lines as monitored by live cell microscopy was not significantly stimulated or inhibited by CG34 (Figure $3 \mathrm{E}-\mathrm{H})$.
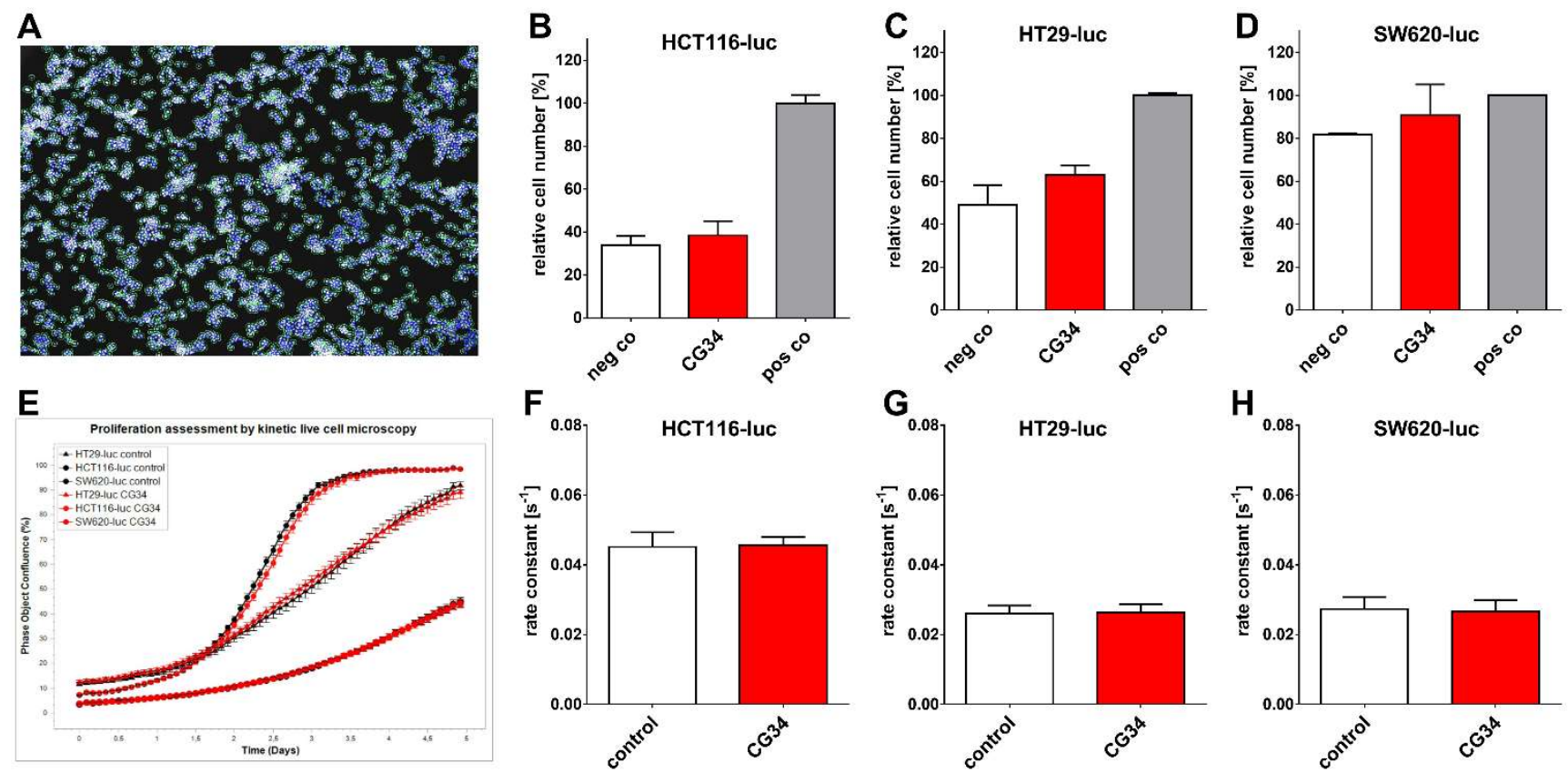

Figure 3. Influence of the chemerin peptide analog CG34 on proliferation of three luciferase-expressing colorectal carcinomacell lines (HCT116-luc, HT29-luc, SW620-luc) in vitro as determined by an endpoint assay (IN Cell high-content analysis of DAPI-stained cell nuclei, upper panel A-D) and by a kinetic assay (confluence measurement using Incucyte live cell microscopy, lower panel E-H).

(A) Exemplary image of the pattern recognition algorithm detecting DAPI-stained nuclei; quantitative results for (B) HCT116-luc; (C)

HT29-luc; (D) SW620-luc. (E) Exemplary result of a kinetic live cell microscopy experiment over five days (black: control cells; red: CG34-treated cells). Quantitative results for (F) HCT116-luc; (G) HT29-luc; (H) SW620-luc. All data represent mean \pm SD of $n=3$ 
Growth of tumor cells in a two-dimensional fashion is only a rough approximation of growth conditions in vivo. Therefore,

another growth assay was applied that allows monitoring proliferation in three-dimensional structures - colonies. Indeed, in a colony formation assay, a concentration of $100 \mathrm{nM}$ of CG34 was able to significantly stimulate growth of all three CRC cell lines. HCT116-luc cells showed a proliferation increase of $10.1 \%(p=0.0118)$, HT29-luc had increased by $9.7 \%$ $(p=0.0124)$, and SW620-luc were stimulated by $16.1 \%(p=0.0045)$.

Apart from proliferation, the ability of tumor cells to migrate is a crucial property influencing the course and kinetics of tumor progression. In a scratch-wound assay quantifying relative changes of the wound density, chemerin receptor stimulation by CG34 at $10 \mu \mathrm{M}$ concentration did not change cell migration in any of the three CRC cell line models versus control conditions (Figure $4 \mathrm{~B}-\mathrm{F})$.

A

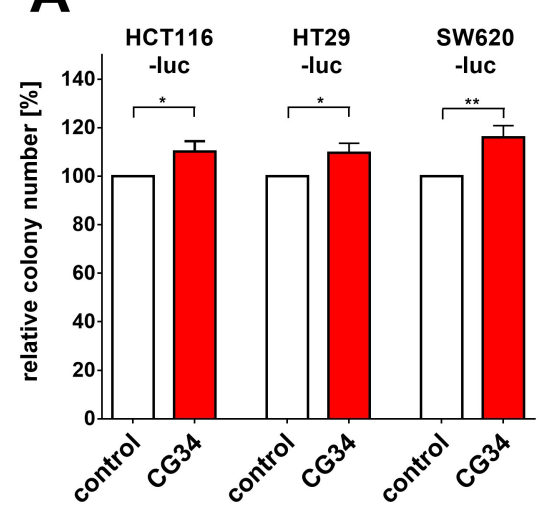

D

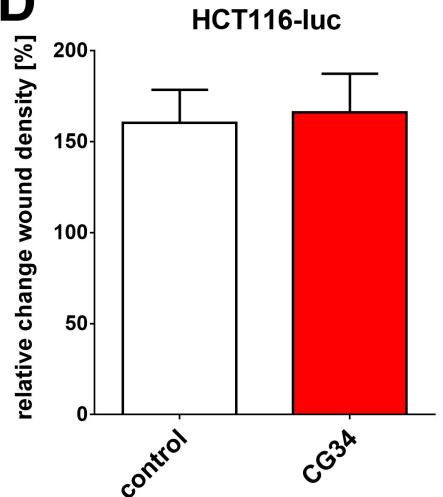

B

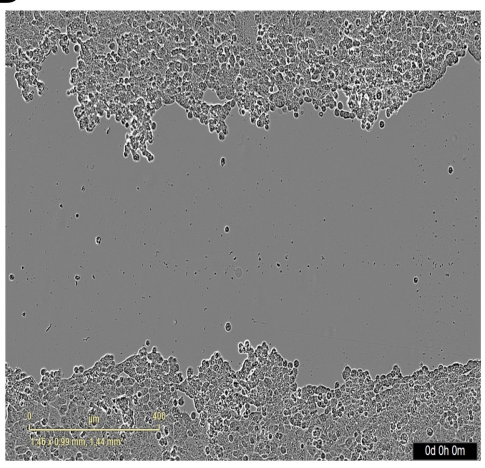

E

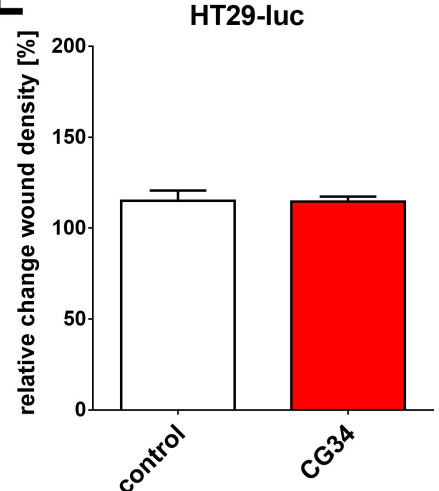

C
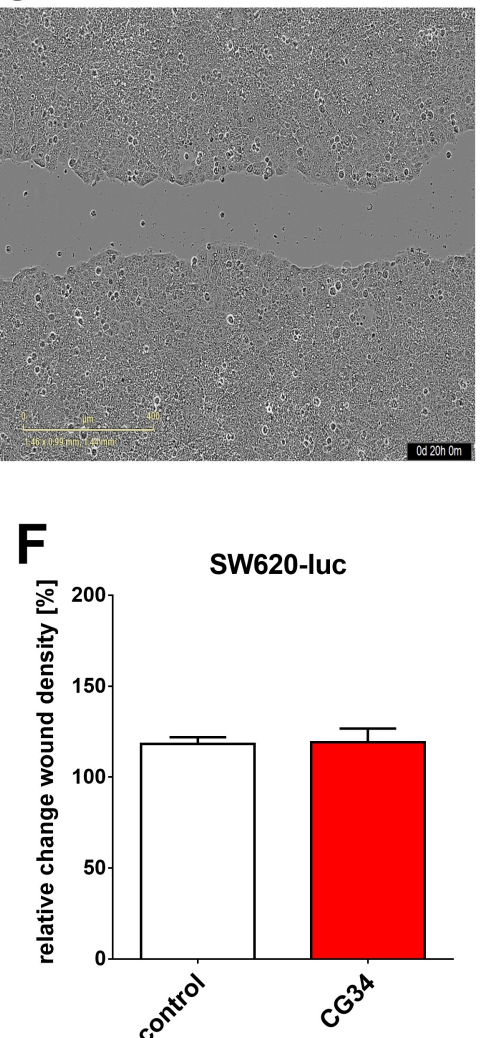

Figure 4. Influence of the chemerin peptide analog CG34 on colony formation and migration of three luciferase-expressing colorectal carcinoma cell lines (HCT116-luc, HT29-luc, SW620-luc). (A) Results of the colony formation assay. Colony formation data represent mean \pm SD of $n=3-4$ independent experiments performed in triplicate. Statistical significance was determined with an unpaired t-test; ${ }^{*} p<0.05,{ }^{* *} p<0.01$. (B) Exemplary image of a migration experiment at the start of treatment (0 hours). (C) Image of the same well as in (B), taken 20 hours later. (D-F) quantitative results of the migration assay: (D) HCT116-luc; (E) HT29-luc; (F) SW620-luc. 
As the in vitro analysis of three CRC cell lines had yielded indications for a potential involvement of chemerin receptor signaling in growth regulation, an in vivo study was designed to address this hypothesis in an animal model. Of the three cell models used in vitro, two were chosen for the animal study: HCT116-luc (low to moderate chemerin receptor ex301 pression, no secretion of the ligand chemerin) and HT29-luc (low to moderate CMKLR1 and GPR expression, moderate chemerin secretion). SW620-luc were excluded, as they endogenously secrete high amounts of the ligand chemerin and any effect of an exogenous chemerin analogue may be obscured. As the two CRC cell lines chosen were of human origin, immunodeficient NMRI nu/nu nude mice were applied to generate subcutaneous tumor xenografts of HCT116luc and HT29-luc cells. Animals were treated by i.p. injection daily, starting from day 8 and ending on day 28 after tumor cell inoculation. After randomization, animals received either a bolus of $50 \mathrm{nmol}$ of the chemerin analog CG34 or water. Both tumor size (caliper measurement) as well as tumor bioluminescence (whole-animal imaging) as a measure of both tumor size/cell number as well as tumor cell viability were monitored on days 1, 7, 15, 21, and 28 of the experiment. From day 15 of the experiment onward, tumor volumes in treatment and control group started to differ for both HCT116luc as well as for HT29-luc xenografts, with tumor volumes in the treatments groups being higher than in the control groups. On day 28 , differences in tumor volumes (mean \pm SD) were found to be statistically significant for HCT116-luc xenografts in the control group $53.9 \pm 52.4 \mathrm{~mm}^{3}$ versus $189 \pm 100 \mathrm{~mm}^{3}$ in the CG34 treatment group ( $\left.p=0.0151\right)$. For HT29-luc xenografts, volumes in the control group $\left(261 \pm 147 \mathrm{~mm}^{3}\right)$ were considerably lower than in the treatment group, though not statistically significant $\left(427 \pm 250 \mathrm{~mm}^{3}, \mathrm{p}=0.1906\right)$ (Figure 5A). 

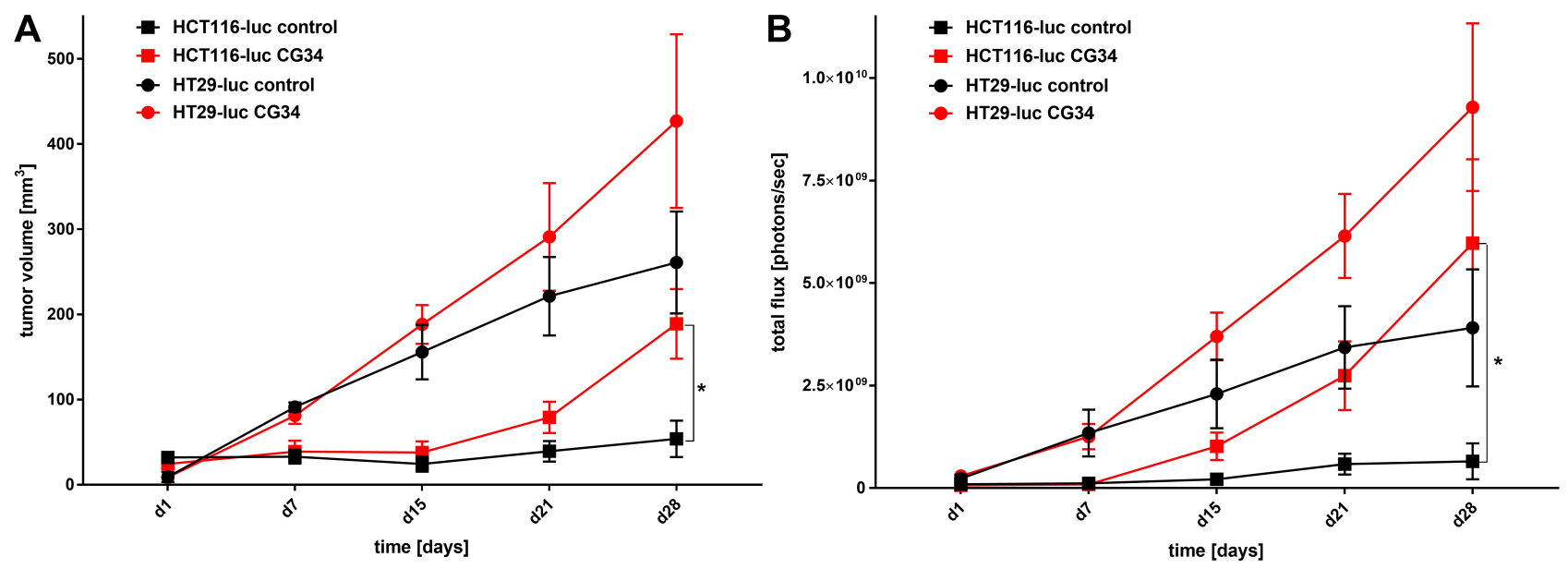

C

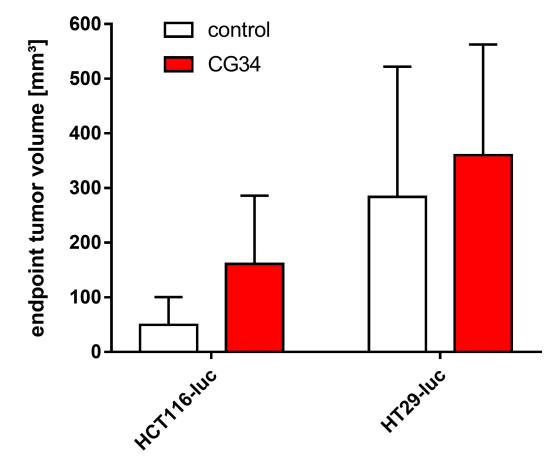

D

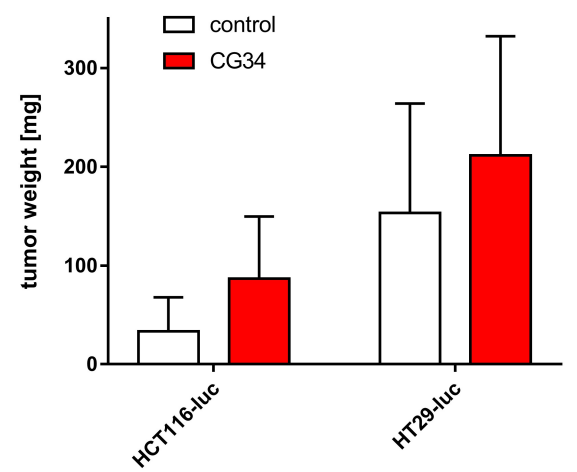

Figure 5. Influence of the chemerin peptide analog CG34 on the growth of two human CRC xenograft mouse models. Five million of

the luciferase-expressing HCT116-luc and HT29-luc cells were used to inoculate female NMRI nu/nu mice. On day 8 after inoculation, daily treatment with i.p. injections of CG34 or water was started in an investigator-blinded fashion; treatment was continued until day 28. (A) Tumor volume measurements performed with a caliper; (B) Bioluminescence measurements performed with a high-sensitivity CCD imager; (C) Volume measurements performed with a caliper at the end of the experiment after mice had been sacrificed; (D)

Tumor weight measurements performed at the end of the experiment using explanted tumors. Volume and bioluminescence data (A,

B) represent mean \pm S.E.M. of $n=6$ tumors per group. Statistical verification was performed using an unpaired t-test or a Mann-

Similarly, bioluminescence intensity evaluated as total flux (photons per second), started to develop differently from day 
At the termination of the experiment, sizes and weights of tumors from sacrificed animals were determined. For both volume and weight, all mean values from tumors in the CG34 treatment group were considerably higher than the control group, even though, due to high variance, the differences lacked statistical significance (Figure 5 C, D).

To investigate a potential involvement of tumor vascularization in growth differences between the groups in the animal study, tissue cryosections from all tumors were prepared and stained immunohistochemically with a marker antibody for blood vessels (CD31). While vessel numbers per area were not different for HCT116-luc xenografts, in HT29-luc xenografts from the CG34 treatment group, they were higher than in the control group (Figure 6 A, C).

As tumor growth is associated with regulation of cellular proliferation, cryosections were also stained with a marker antibody for mitotic activity (Ki67). In this experiment, Ki67-positive cell numbers per area (mean \pm SD) were significantly higher in HCT116-luc xenografts from the treatment group (45.84 \pm 9.29$)$ than from the control $(38.37 \pm 9.99 ; p=0.362)$. In the HT29-luc groups, no significant difference was found Figure 6 B, D).

A

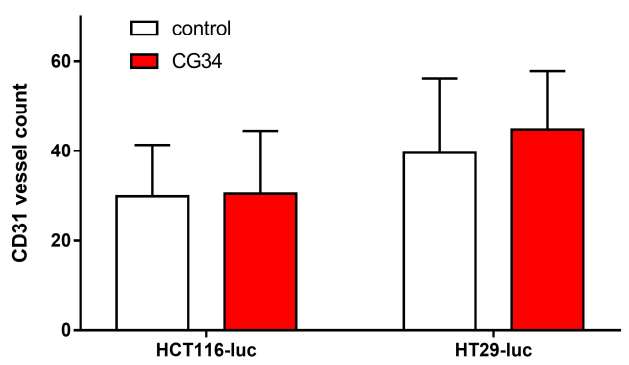

C

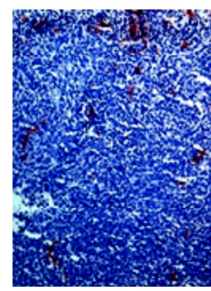

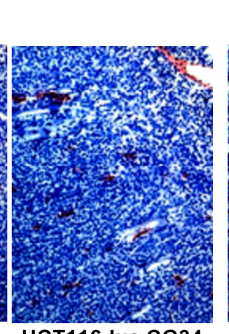

HCT116-luc CG34
HCT116-luc co

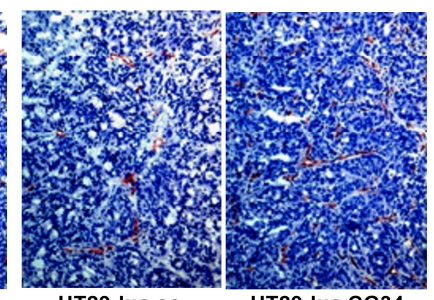

HT29-luc co
B

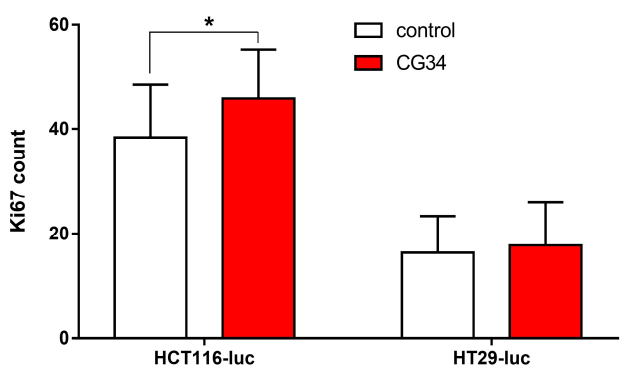

D

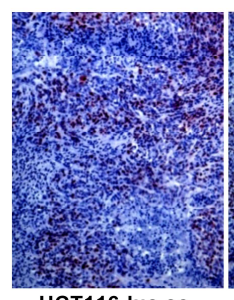

HCT116-luc co

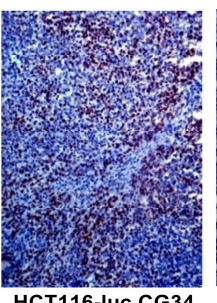

HCT116-luc CG34

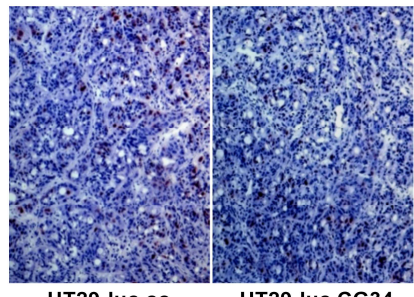

HT29-luc co
346

347

Figure 6. Immunohistological analysis and quantitation of vessel density (CD31 staining; A, C) and proliferative activity (Ki67 staining; B, D) in sections of xenograft tumors after the treatment study (Fig. 5). Quantitative data (A, B) represent mean \pm SD of $n=15-24$ analyzed microscopic imaging fields. Statistical testing was performed using an unpaired t-test; * $\mathrm{p}<0.05$.

\section{Discussion}

Chemerin has been demonstrated to be bound by and to activate two heptahelical plasma membrane receptors, CMKLR1 and GPR1, while a third, CCRL2, binds chemerin and is believed to pass on the ligand to the other receptors $[1,2]$. The expression of the three receptors has been revealed to some degree in healthy organs and tissues, where they act to transmit the various chemerin-mediated physiological functions from immune cell attraction to metabolic 
bioRxiv preprint doi: https://doi.org/10.1101/2021.11.19.469262; this version posted November 20, 2021. The copyright holder for this preprint (which was not certified by peer review) is the author/funder, who has granted bioRxiv a license to display the preprint in perpetuity. It is made available under aCC-BY-NC-ND 4.0 International license.

regulation [32]. In cancer, however, expression of CMKLR1, GPR1 and CCRL2 has not been studied to great extent. In CRC, CMKLR1 was discovered at higher abundance in CRC tumor tissues than in margins [30]. In addition, CMKLR1 was found to correlate with GDF-15 and VEGF-A levels in CRC tumor-free margin [33]. Our own previous report revealed CMKLR1 to be highly expressed in the breast cancer cell line DU4475 [31]. The current study shows quantitative mRNA abundance data for five CRC and 35 other cell lines, revealing complex and differential expression patterns that do not point to a particular, specific role for any of the receptors in one of the tumor entities under investigation. Rather, CMKLR1 and GPR1 expression levels vary across many orders of magnitude for most tumor types, while CCRL2 is completely missing from seven of the tested cancer cell lines (Fig. 1). In CRC cell lines, the three cell lines HCT116, HT29 and SW620 represent the different expression patterns and were therefore selected for subsequent experiments.

In contrast to the receptors, the ligand chemerin has been extensively characterized with regard to tumor expression and serum levels $[18,19]$. Most research, however, has been focusing on immune cells, cancer-associated fibroblasts and other components of the tumor microenvironment as sources of chemerin secretion. This study demonstrated significant chemerin mRNA levels in cancer cell lines from brain, stomach, pancreas and colon (Fig. 1). Relevant chemerin concentrations in the supernatant of cultured cells, however, were only detected in two glioblastoma as well as in three CRC cell lines, potentially suggesting a role of tumor cell-produced chemerin in the biology of these cancer types. Tumor cells as a source of chemerin so far have been studied in the context of artificial overexpression or intratumoral injection of chemerin [20,34], while secretion from cell lines has not been studied so far, to the best of our knowledge.

Several tumor types so far have been identified to feature a direct chemerin action on tumor cell proliferation and tumor growth, which may be either stimulating or inhibiting [35]. In gastric cancer, e.g., chemerin was observed to stimulate carcinogenesis by inducing phosphorylation of p38 and ERK 1/2 MAPKs. This study did not find a clear stimulatory or inhibitory action of the chemerin analog CG34 on the three CRC cell lines HCT116-luc, HT29-luc, and SW620-luc when grown in a two-dimensional monolayer. However, when these cells were monitored for three-dimensional colony formation, CG34 significantly promoted growth in all three (Fig. 4 A). While these results may appear contradictory, it is conceivable that the growth-enhancing effect of the chemerin analog depends on signaling only active in a three-dimensional cell context. It has been established that cells adapt to their environment by reacting to local signals, which in turn results in changes in cell proliferation and other physiological functions [36]. Still, one limitation of this study is the lack of a mechanistic explanation for the differential regulation by chemerin in $2 \mathrm{D}$ versus $3 \mathrm{D}$ cultures. Further studies will have to elucidate the signaling processes involved in chemerin-induced enhancement of CRC cell colony formation.

Similar to its action on CRC cell colonies, CG34 clearly stimulated the growth of HCT116-luc as well as HT29-luc xenografts in a subcutaneous nude mouse model, as measured by tumor volume and bioluminescence intensity (Fig. 
5). Statistical significance was found for differences between CG34-treated and control group in HCT116-luc xenografts, while a high variance in values for HT29 precluded a statistically significant result. Similarly, tumor volume and weight were considerably increased. These results are indicative of a strong stimulatory function of chemerin signaling in CRC growth regulation. One of the potential influences of chemerin in regulation tumor growth that has been widely studied is its impact on the formation of new vessels [18]. Ex-vivo analysis of tumors from the animal study demonstrated only a minor, non-significant enhancement of vessel density in HT29-luc xenografts of the CG34 treatment group versus control (Fig. 6 A, C).

Though a correlation of chemerin levels and CRC risk has been established [27-29], a regulatory effect has not been 398 reported in the literature to date. Even though this study, hence, provides the first report of a chemerin agonist stimulating CRC in an animal model, it has a number of limitations. As the chemerin analog was administered systemically via a vein, this analysis cannot distinguish direct effects of CG34 on tumor cells from indirect regulation via immune cells, cancer-associated fibroblasts or other cells of the tumor microenvironment. Indeed, indirect such indirect effects dominate in most reports on chemerin effects in cancers $[18,19,35]$. It is, however, intriguing that both in in vitro colony formation as well as in the animal study, CG34 led to increased growth, potentially suggesting a direct effect of the analog on CRC cells. This is also supported by the detection of a statistically significant increase in the mitosis marker Ki67 in HCT116-luc xenografts of the CG34 treatment group versus tissues from the control group (Fig. 6 B, D). Further studies will have to provide confirmation as well as clues about the exact molecular nature of this tumor-promoting activity.

\section{Conclusions}

The chemerin analog CG34 proved to stimulate colony formation as well as xenograft growth in two mouse models of colorectal carcinoma, with intratumor Ki67 cells increased in one of these models. These results strongly indicate a regulating, stimulatory effect of chemerin receptor signaling in colorectal carcinoma.

Author Contributions: Conceptualization, P.S. and C.G.; methodology, J.F.-K., S.R. and Q.S.; formal analysis, J.F.-K., P.S. and 
Institutional Review Board Statement: Animal care followed federal, local and institutional guidelines and all experiments were approved by local animal welfare authorities.

Data Availability Statement: Numerical, machine-readable data for this study have been deposited in an open data repository for public access: $\underline{\text { http://doi.org/10.5281/zenodo.5710900 }}$

Acknowledgments: The authors wish to thank Annett Rexin, Ines Eichhorn and Yvonne Giesecke for excellent technical support. The authors acknowledge support by the Molecular Cancer Research Center (MKFZ), Charité - Universitätsmedizin Berlin, 13353 Berlin, Germany.

Conflicts of Interest: The authors declare no conflict of interest. The funders had no role in the design of the study; in the collection, analyses, or interpretation of data; in the writing of the manuscript, or in the decision to publish the results.

\section{References}

1. Rourke, J.L.; Dranse, H.J.; Sinal, C.J. Towards an integrative approach to understanding the role of chemerin in human health and disease. Obes. Rev. 2013, 14, 245-262, doi:10.1111/obr.12009.

2. Helfer, G.; Wu, Q.F. Chemerin: A multifaceted adipokine involved in metabolic disorders. J. Endocrinol. 2018, 238, R79-R94, doi:10.1530/JOE-18-0174.

3. Ernst, M.C.; Haidl, I.D.; Zuńĩga, L.A.; Dranse, H.J.; Rourke, J.L.; Zabel, B.A.; Butcher, E.C.; Sinal, C.J. Disruption of the chemokine-like receptor-1 (CMKLR1) gene is associated with reduced adiposity and glucose intolerance. Endocrinology 2012, 153, 672-682, doi:10.1210/en.2011-1490.

4. Ernst, M.C.; Issa, M.; Goralski, K.B.; Sinal, C.J. Chemerin exacerbates glucose intolerance in mouse models of obesity and diabetes. Endocrinology 2010, 151, 1998-2007, doi:10.1210/en.2009-1098.

5. Bozaoglu, K.; Bolton, K.; McMillan, J.; Zimmet, P.; Jowett, J.; Collier, G.; Walder, K.; Segal, D. Chemerin is a novel adipokine associated with obesity and metabolic syndrome. Endocrinology 2007, 148, 4687-4694, doi:10.1210/en.2007-0175.

6. Ress, C.; Tschoner, A.; Engl, J.; Klaus, A.; Tilg, H.; Ebenbichler, C.F.; Patsch, J.R.; Kaser, S. Effect of bariatric surgery on circulating chemerin levels. Eur. J. Clin. Invest. 2010, 40, 277-280, doi:10.1111/j.1365-2362.2010.02255.x.

7. Wittamer, V.; Franssen, J.D.; Vulcano, M.; Mirjolet, J.F.; Le Poul, E.; Migeotte, I.; Brézillon, S.; Tyldesley, R.; Blanpain, C.; Detheux, M.; et al. Specific recruitment of antigen-presenting cells by chemerin, a novel processed ligand from human inflammatory fluids. J. Exp. Med. 2003, 198, 977-985, doi:10.1084/jem.20030382.

8. Zabel, B.A.; Silverio, A.M.; Butcher, E.C. Chemokine-Like Receptor 1 Expression and Chemerin-Directed Chemotaxis Distinguish Plasmacytoid from Myeloid Dendritic Cells in Human Blood. J. Immunol. 2005, 174, 244-251, doi:10.4049/jimmunol.174.1.244.

9. Mitchell, D.; Chintala, S.; Dey, M. Plasmacytoid dendritic cell in immunity and cancer. J. Neuroimmunol. 2018, 322, 63-73, doi:10.1016/j.jneuroim.2018.06.012.

10. Jaworek, J.; Szklarczyk, J.; Kot, M.; Góralska, M.; Jaworek, A.; Bonior, J.; Leja-Szpak, A.; Nawrot-Porąbka, K.; LinkLenczowski, P.; Ceranowicz, P.; et al. Chemerin alleviates acute pancreatitis in the rat thorough modulation of NF-KB signal. Pancreatology 2019, 19, 401-408, doi:10.1016/j.pan.2019.02.005.

11. Dimitriadis, G.K.; Kaur, J.; Adya, R.; Miras, A.D.; Mattu, H.S.; Hattersley, J.G.; Kaltsas, G.; Tan, B.K.; Randeva, H.S. Chemerin induces endothelial cell inflammation: Activation of nuclear factor-kappa beta and monocyte-endothelial adhesion. Oncotarget 2018, 9, 16678-16690, doi:10.18632/oncotarget.24659.

12. Shang, J.; Wang, L.; Zhang, Y.; Zhang, S.; Ning, L.; Zhao, J.; Cheng, G.; Liu, D.; Xiao, J.; Zhao, Z. Chemerin/ChemR23 axis promotes inflammation of glomerular endothelial cells in diabetic nephropathy. J. Cell. Mol. Med. 2019, 23, 3417-3428, 
doi:10.1111/jcmm.14237.

13. John, H.; Hierer, J.; Haas, O.; Forssmann, W.G. Quantification of angiotensin-converting-enzyme-mediated degradation of human chemerin 145-154 in plasma by matrix-assisted laser desorption/ionization-time-of-flight mass spectrometry. Anal. Biochem. 2007, 362, 117-125, doi:10.1016/j.ab.2006.12.001.

14. Huang, H.; Hu, L.; Lin, J.; Zhu, X.; Cui, W.; Xu, W. Effect of fosinopril on chemerin and VEGF expression in diabetic nephropathy rats. Int. J. Clin. Exp. Pathol. 2015, 8, 11470-11474.

15. Buechler, C.; Feder, S.; Haberl, E.M.; Aslanidis, C. Chemerin isoforms and activity in obesity. Int. J. Mol. Sci. 2019, 20, doi:10.3390/ijms20051128.

16. Ferland, D.J.; Watts, S.W. Chemerin: A comprehensive review elucidating the need for cardiovascular research. Pharmacol. Res. 2015, 99, 351-361, doi:10.1016/J.PHRS.2015.07.018.

17. Ferland, D.J.; Mullick, A.E.; Watts, S.W. Chemerin as a Driver of Hypertension: A Consideration. Am. J. Hypertens. 2020, 33, 975-986, doi:10.1093/AJH/HPAA084.

18. Goralski, K.B.; Jackson, A.E.; McKeown, B.T.; Sinal, C.J. More Than an Adipokine: The Complex Roles of Chemerin Signaling in Cancer. Int. J. Mol. Sci. 2019, 20, 4778, doi:10.3390/ijms20194778.

19. Treeck, O.; Buechler, C.; Ortmann, O. Chemerin and cancer. Int. J. Mol. Sci. 2019, 20, doi:10.3390/ijms20153750.

20. Pachynski, R.K.; Zabel, B.A.; Kohrt, H.E.; Tejeda, N.M.; Monnier, J.; Swanson, C.D.; Holzer, A.K.; Gentles, A.J.; Sperinde, G. V.; Edalati, A.; et al. The chemoattractant chemerin suppresses melanoma by recruiting natural killer cell antitumor defenses. J. Exp. Med. 2012, 209, 1427-1435, doi:10.1084/jem.20112124.

21. Dubois-Vedrenne, I.; De Henau, O.; Robert, V.; Langa, F.; Javary, J.; Al Delbany, D.; Vosters, O.; Angelats-Canals, E.; Vernimmen, M.; Luangsay, S.; et al. Expression of Bioactive Chemerin by Keratinocytes Inhibits Late Stages of Tumor Development in a Chemical Model of Skin Carcinogenesis. Front. Oncol. 2019, 9, 1-15, doi:10.3389/fonc.2019.01253.

22. Lin, Y.; Yang, X.; Liu, W.; Li, B.; Yin, W.; Shi, Y.; He, R. Chemerin has a protective role in hepatocellular carcinoma by inhibiting the expression of IL-6 and GM-CSF and MDSC accumulation. Oncogene 2017, 36, 3599-3608, doi:10.1038/onc.2016.516.

23. Li, J.J.; Yin, H.K.; Guan, D.X.; Zhao, J.S.; Feng, Y.X.; Deng, Y.Z.; Wang, X.; Li, N.; Wang, X.F.; Cheng, S.Q.; et al. Chemerin suppresses hepatocellular carcinoma metastasis through CMKLR1-PTEN-Akt axis. Br. J. Cancer 2018, 118, 1337-1348, doi:10.1038/s41416-018-0077-y.

24. Wei Lin, Yan-Ling Chen, Lei Jiang, J.-K.C. Reduced expression of chemerin is associated with a poor prognosis and a lowed infiltration of both dendritic cells and natural killer cells in human hepatocellular carcinoma. Clin. Lab. 2011, 57, 879-885, doi:Clin.Lab.2011;57:879-885.

25. Tümmler, C.; Snapkov, I.; Wickström, M.; Moens, U.; Ljungblad, L.; Elfman, L.H.M.; Winberg, J.O.; Kogner, P.; Johnsen, J.I.; Sveinbjørnsson, B. Inhibition of chemerin/CMKLR1 axis in neuroblastoma cells reduces clonogenicity and cell viability in vitro and impairs tumor growth in vivo. Oncotarget 2017, 8, 95135-95151, doi:10.18632/oncotarget.19619.

26. Dinesh Kumar, J.; Aolymat, I.; Tiszlavicz, L.; Reisz, Z.; Garalla, H.M.; Beynon, R.; Simpson, D.; Dockray, G.J.; Varro, A. Chemerin acts via CMKLR1 and GPR1 to stimulate migration and invasion of gastric cancer cells: Putative role of decreased TIMP-1 and TIMP-2. Oncotarget 2019, 10, 98-112, doi:10.18632/oncotarget.26414.

27. Eichelmann, F.; Schulze, M.B.; Wittenbecher, C.; Menzel, J.; Weikert, C.; di Giuseppe, R.; Biemann, R.; Isermann, B.; Fritsche, A.; Boeing, H.; et al. Association of Chemerin Plasma Concentration With Risk of Colorectal Cancer. JAMA Netw. open 2019, 2, e190896, doi:10.1001/jamanetworkopen.2019.0896.

28. Erdogan, S.; Yilmaz, F.M.; Yazici, O.; Yozgat, A.; Sezer, S.; Ozdemir, N.; Uysal, S.; Purnak, T.; Sendur, M.A.; Ozaslan, E. Inflammation and chemerin in colorectal cancer. Tumor Biol. 2016, 37, 6337-6342, doi:10.1007/s13277-015-4483-y.

29. 
30. Kiczmer, P.; Seńkowska, A.P.; Kula, A.; Dawidowicz, M.; Strzelczyk, J.K.; Zajdel, E.N.; Walkiewicz, K.; Waniczek, D.; Ostrowska, Z.; Świętochowska, E. Assessment of CMKLR1 level in colorectal cancer and its correlation with angiogenic markers. Exp. Mol. Pathol. 2020, 113, 1-5, doi:10.1016/j.yexmp.2020.104377.

31. Erdmann, S.; Niederstadt, L.; Koziolek, E.J.; Gómez, J.D.C.; Prasad, S.; Wagener, A.; von Hacht, J.L.; Reinicke, S.; Exner, S.; Bandholtz, S.; et al. CMKLR1-targeting peptide tracers for PET/MR imaging of breast cancer. Theranostics 2019, 9, 67196733, doi:10.7150/thno.34857.

507 508 509 510 511

32. Yoshimura, T.; Oppenheim, J.J. Chemokine-like receptor 1 (CMKLR1) and chemokine (C-C motif) receptor-like 2 (CCRL2); Two multifunctional receptors with unusual properties. Exp. Cell Res. 2010, 1, 1-11, doi:10.1016/j.yexcr.2010.10.023.

33. Mielcarska, S.; Stopińska, K.; Dawidowicz, M.; Kula, A.; Kiczmer, P.; Seńkowska, A.P.; Zajdel, E.N.; Walkiewicz, K.; Waniczek, D.; Świętochowska, E. GDF-15 Level Correlates with CMKLR1 and VEGF-A in Tumor-free Margin in Colorectal Cancer. Curr. Med. Sci. 2021413 2021, 41, 522-528, doi:10.1007/S11596-021-2335-0.

34. Pachynski, R.K.; Wang, P.; Salazar, N.; Zheng, Y.; Nease, L.; Rosalez, J.; Leong, W.I.; Virdi, G.; Rennier, K.; Shin, W.J.; et al. Chemerin suppresses breast cancer growth by recruiting immune effector cells into the tumor microenvironment. Front. Immunol. 2019, 10, 983, doi:10.3389/FIMMU.2019.00983/BIBTEX.

35. Shin, W.J.; Pachynski, R.K. Chemerin Modulation of Tumor Growth: Potential Clinical Applications in Cancer. Discov. Med. 2018, 26, 31-37.

36. Schiavone, W.A.; Baker, C.; Prasad, S.K. Journal of Cardiovascular Magnetic Imaging myocardial carcinoid with T2-STIR CMR. J. Cardiovasc. Magn. Reson. 2008, 5, 1-5, doi:10.1186/1532-429X-10-Received. 


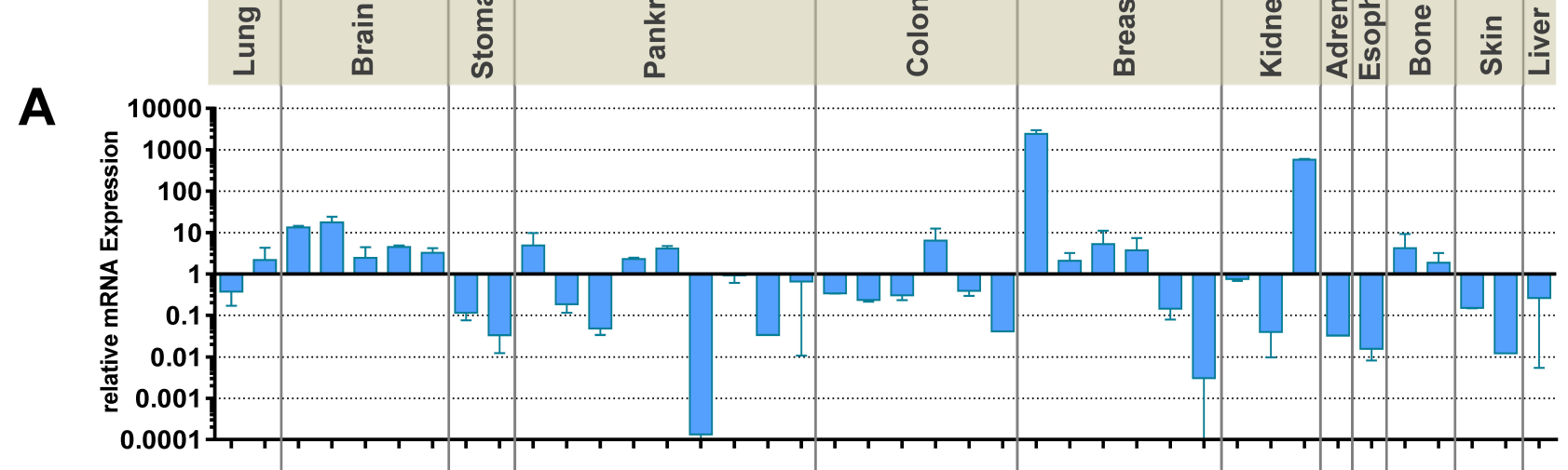

B

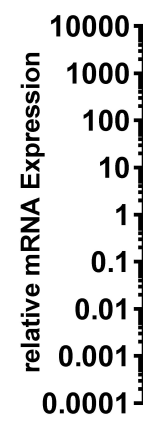

C

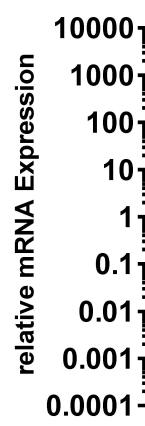

D

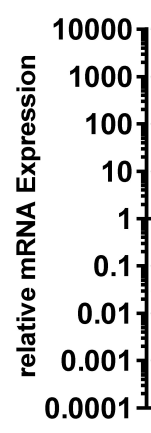

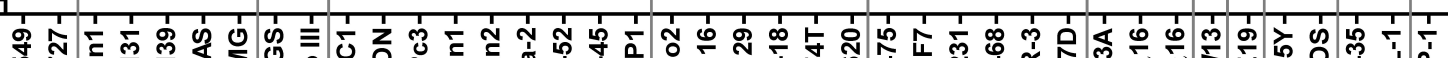

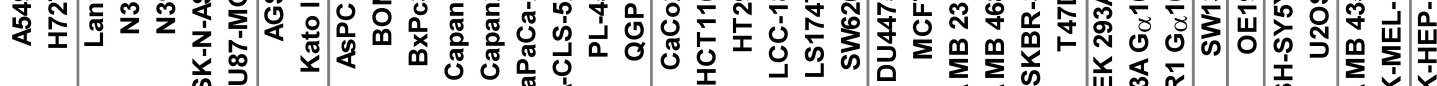

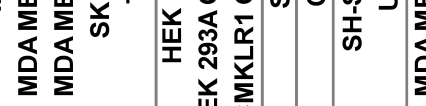



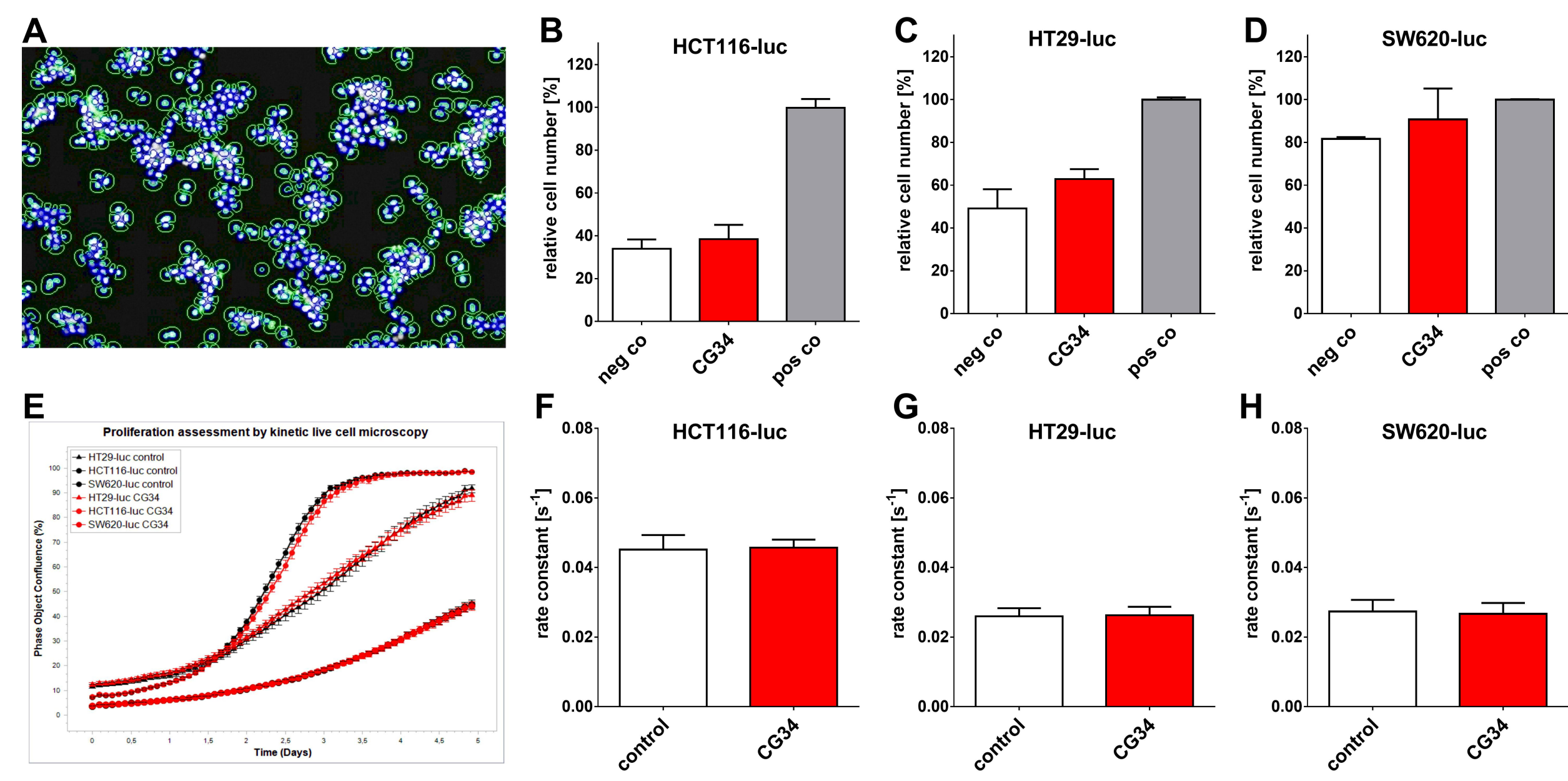
$\begin{array}{ll}\text { A } & \text { B }\end{array}$
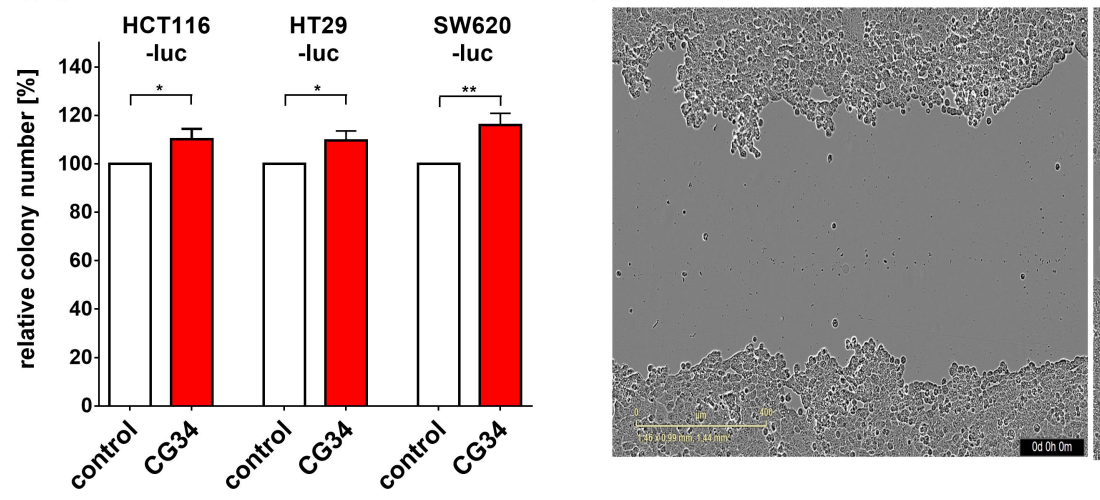

C
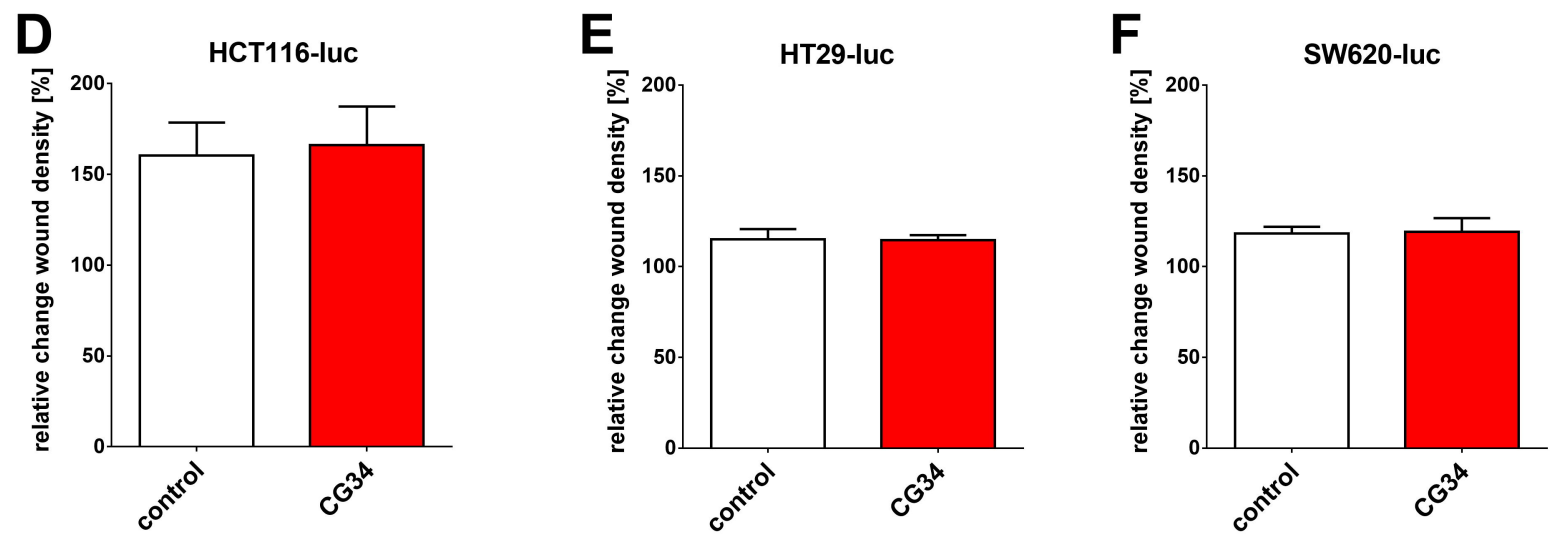

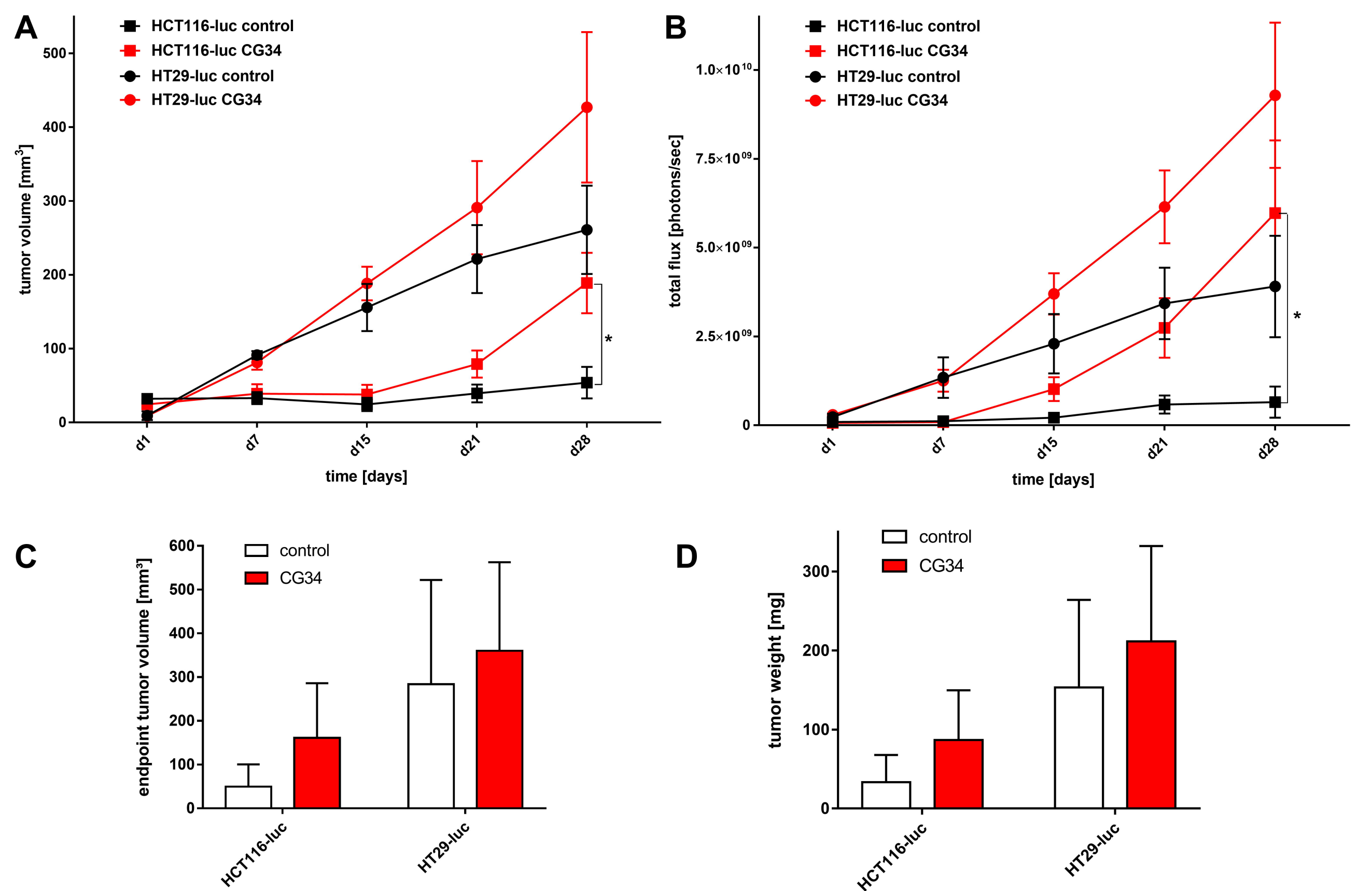


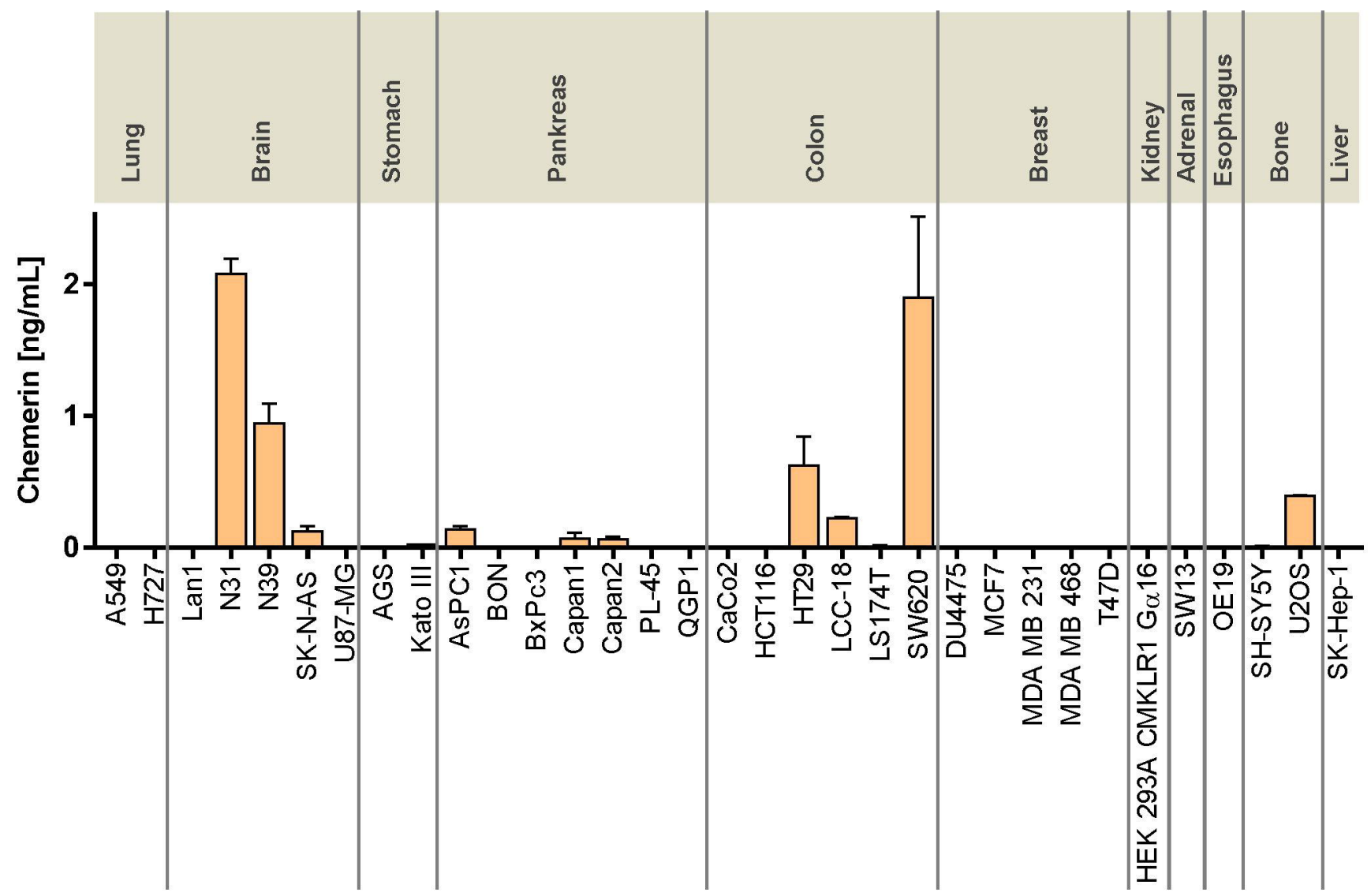


